\title{
The use of conscious sedation in elective external direct current cardioversion: a single centre experience
}

\author{
Ronstan Lobo, Thomas Kiernan \\ University Hospital Limerick, Republic of Ireland
}

\begin{abstract}
External direct current (DC) cardioversion is a commonly used method of terminating atrial arrhythmias. The chance of procedural success is inversely related to the duration of the arrhythmia. In many hospitals, the procedure is carried out under general anaesthesia, necessitating the presence of anaesthetic as well as medical staff. Frequently, it may be difficult to coordinate the availability of the two teams, causing delays to each patient, waste of staff time, an inefficient service and increased costs. The primary aim of the study was to determine the safety and tolerability of conscious sedation using intravenous midazolam in elective external DC cardioversion of patients with atrial flutter or atrial fibrillation attending University Hospital Limerick, Ireland.

Patients who were electively admitted for DC cardioversion for atrial fibrillation or atrial flutter were recruited. A pre-defined sedation protocol using intravenous midazolam was used. The midazolam was given by the cardiology registrar without the presence of an anaesthetist. DC cardioversion was then performed according to the hospital protocol. Any complications arising during the procedure were noted. Prior to discharge, patients were given a questionnaire to determine their awareness of the procedure and if they would have the procedure done again in the future if needed.
\end{abstract}

A total of 100 patients were recruited. The median number of shocks was one (highest number of shocks being four). The median dose of midazolam was $7.5 \mathrm{mg}$, with the highest dose being $20 \mathrm{mg}$. All of patients surveyed were not aware of the shock that was administered to them. All of the patients surveyed were happy with the service and would be happy to return for a repeat cardioversion in the future if required.

The use of conscious sedation for DC cardioversion of patients with atrial fibrillation / atrial flutter was found to be safe and tolerable.

\section{Problem}

External direct current (DC) cardioversion is a commonly used method of terminating atrial arrhythmias. The chance of procedural success is inversely related to the duration of the arrhythmia. Rapid patient turnover is therefore of key importance in managing this condition. In many hospitals, the procedure is carried out under general anaesthesia, necessitating the presence of anaesthetic as well as cardiology staff. Frequently, it may be difficult to coordinate the availability of the two teams, causing delays to each patient, waste of staff time, an inefficient service and increased costs.

The concerns about the use of midazolam in conscious sedation arise from two factors. The first factor is the difficulty in controlling the sedation using midazolam when compared to general anaesthesia. The second factor is the belief that midazolam use in conscious sedation does not result in adequate anaesthesia as in many cases, the patients awake and sometimes appear to be in pain when the shock is delivered. This study aims to formally assess both factors.

\section{Background}

There are studies that report successful cardioversions using conscious sedation which is safe and tolerable for patients.[1-5] The primary aim of the study was to determine the safety and tolerability of conscious sedation using intravenous midazolam in elective external DC cardioversion of patients with atrial fibrillation or atrial flutter attending University Hospital Limerick, Ireland. The simplified protocol was performed by a cardiology registrar in the coronary care unit without the presence of an anaesthetist.

\section{Baseline measurement}

Many hospitals throughout the Republic of Ireland and the United Kingdom perform elective external DC cardioversion under general anaesthesia. Our centre has been using conscious sedation for DC cardioversion since the procedures were first performed here. While there have been no adverse events noted, no formal study on the tolerability based on patient experience was ever conducted.

\section{Design}

This was an observational study with patient feedback via the use of a questionnaire prior to patient discharge.

Patients who were electively admitted for DC cardioversion for atrial fibrillation or atrial flutter were recruited. A pre-defined sedation protocol using intravenous midazolam was used. Midazolam was given as a $5 \mathrm{mg}$ bolus followed by $2.5 \mathrm{mg}$ titrated doses every two 
minutes until the patient is adequately sedated. Adequate sedation is determined by loss of response to gentle verbal stimulus or tactile stimulus. DC cardioversion was then performed according to the hospital protocol. All cardioversions were performed using a biphasic external cardioverter/defibrillator. Top up doses of $1 \mathrm{mg}$ to $2.5 \mathrm{mg}$ were given in between shocks if the patient required it to ensure adequate sedation was achieved.

Complications arising during the procedure were noted. Patients were then monitored for four hours prior to discharge. Prior to discharge, patients were given a questionnaire to complete. The questionnaire included questions on the patient's awareness of the procedure and if they would have the procedure done again in the future if needed.

\section{Strategy}

Plan: Measure safety and tolerability of conscious sedation use in elective external direct current cardioversion for atrial fibrillation / atrial flutter.

Do: Record complications resulting from use of conscious sedation during procedure. Record patient tolerability of the procedure by use of a questionnaire.

Study: Analyse results from the data collected.

Act: Publish results to help affect change.

\section{Results}

A total of 100 patients were recruited (table 1). There were 89 successful cardioversions (cardioversions that achieved normal sinus rhythm). The median number of shocks was one (highest number of shocks being four). The median dose of midazolam was $7.5 \mathrm{mg}$, with the highest dose being $20 \mathrm{mg}$. There were four patients that required midazolam reversal using flumazenil; two patients because of high doses of midazolam used (more than 10 $\mathrm{mg}$ ), one patient because of an atypical response to sedation, and one patient whose oxygen saturation levels dropped postprocedure. All four of these patients were discharged home the same day.

The questionnaire results revealed that all $100 \%$ of the patients were not aware of the shock that was administered to them. All $100 \%$ of the patients surveyed were happy with the service and would be happy to return for a repeat cardioversion in the future if required.

See supplementary file: ds5204.PNG - "Table"

\section{Lessons and limitations}

We learned that the use of conscious sedation in elective DC cardioversion is safe and tolerable. Even though it may seem cruel to use conscious sedation as the patient may awake or appear to be in pain when the shock is delivered, our study showed that $100 \%$ of our patients did not remember the procedure by 4 hours postprocedure and were happy to return for a repeat procedure if indicated. The safety profile of judicious use of intravenous midazolam was also demonstrated in this study.

The limitations of this study is a relatively small sample size. Also, delayed recall was not accessed in this study although it was shown in a previously published study that midazolam use in DC cardioversion affected delayed recall resulting in a more complete amnestic effect(6).

\section{Conclusion}

The use of conscious sedation for DC cardioversion of patients with atrial fibrillation or atrial flutter was found to be safe and comfortable, with $100 \%$ of the patients surveyed not being aware of the shock that was administered to them and $100 \%$ of the patients surveyed being happy with the service and would be happy to return for a repeat cardioversion in the future if required.

Hospitals that are still using general anaesthesia for patients undergoing DC cardioversion for atrial fibrillation / atrial flutter should consider switching to the use of conscious sedation. This can improve efficiency, cut costs, and reduce waiting times for patients, resulting in improved clinical outcomes.

\section{References}

1. Notarstefano $P$, Pratola $C$, Toselli $T$, Baldo E, Ferrari R. Sedation with midazolam for electrical cardioversion. Pacing Clin Electrophysiol 2007; 30(5): 608-11.

2. Mennuni M, Bianconi L, Antonicoli S, Frongillo D, Molle G, Rossi P, Benturini E, Toscano S. Fast cardiologistadministered midazolam for electrical cardioversion of atrial fibrillation. J Cardiovasc Med (Hagerstown) 2007; 8(3): 176-80.

3. Hubner PJ, Gupta S, McClellan I. Simplified cardioversion service with intravenous midazolam. Heart 2004; 90(12): 1447-9.

4. Paipancholia R, Sentinella L, Lynch M. Role of conscious sedation for external cardioversion. Heart 2001; 86: 571-2.

5. Pugh PJ, Spurrell P, Kamalvand K, et al. Sedation by physician with diazepam for DC cardioversion of atrial arrhythmias. Heart 2001; 86:572-3.

6. Mitchell AR, Chalil S, Boodhoo L, Bordoli G, Patel N, Sulke N. Diazepam or midazolam for external DC cardioversion (the DORM Study). Europace 2003; 5(4): 391-5.

\section{Declaration of interests}

Nothing to declare.

\section{Acknowledgements}

Cardiology nurses of the coronary care unit, University Hospital Limerick. 


\section{Ethical approval}

Ethics approval from this study was obtained from the local research and ethics committee. 